\section{IMPACT OF OCCUPATION ON BLOOD LEAD LEVELS IN PREGNANT WOMEN}

Osmel La Llave Leon, José Manuel Salas Pacheco, Sergio Estrada Martínez, Ada Sandoval Carrillo, Angélica Ma Lechuga Quiñones, Eloísa Esquivel Rodríguez, Gonzalo García Vargas. Universidad Juárez Del Estado de Durango, Durango, Mexico

\subsection{6/oemed-2014-102362.286}

Objectives To examine the relationship between occupation and blood lead levels in pregnant women of Durango, Mexico. Method A cross sectional study was conducted with 299 pregnant women. Information on occupation, risk factors and sociodemographic data was collected by means of a structured questionnaire. Blood lead concentration was tested by graphite furnace spectrometry. Women were divided into three groups according to occupation: working in places with potential source of lead exposure (exposed group), working in places without lead exposure (control group I), and non-working women (control group II). The $\mathrm{X}^{2}$ test was used to assess statistical differences between the groups, and one way ANOVA was applied for comparisons. Logistic regression was performed using blood lead $<5 \mu \mathrm{g} / \mathrm{dL}$ or $\geq 5 \mu \mathrm{g} / \mathrm{dL}$ as dependent variable, and ajdusted for jurisdiction, income, gestational age, and abortions.

Results Only 24(8\%) women worked in places with potential source of lead exposure, $47(15.7 \%)$ worked in other places, and $228(76.3 \%)$ did not have a remunerated job. Mean blood lead concentration in the study sample was $2.79 \mu \mathrm{g} / \mathrm{dL}$. However, blood lead $\geq 5 \mu \mathrm{g} / \mathrm{dL}$ accounted for $25 \%$ of exposed women, $2.1 \%$ of control group I, and $6 \%$ of control group II $\left(\mathrm{X}^{2}=\right.$ 13.04; p .001). Mean blood lead level was $4.24 \mu \mathrm{g} / \mathrm{dL}$ in the exposed group, $2.31 \mu \mathrm{g} / \mathrm{dL}$ in the control group I, and $2.74 \mu \mathrm{g} /$ $\mathrm{dL}$ in the control group $\mathrm{II}$; those differences were statistically significant (0.001). Logistic regression confirmed that blood lead $\geq 5 \mu \mathrm{g} / \mathrm{dL}$ is associated with occupational exposure $(\mathrm{p}=0.036)$. Conclusions Our findings suggest that surveillance for occupational exposure to prevent health damages during pregnancy is needed.

\section{SCREENING AND DISABILITY PREVENTION FOR MUSCULOSKELETAL DISORDERS OF HIGH-TECH INDUSTRY WORKERS IN TAIWAN}

Ya-Yuan Hsu. Institute of Occupational Safety and Health, Taipei, Taiwan

10.1136/oemed-2014-102362.287

Objectives In Taiwan, 40-60\% of the working population is affected by musculoskelet al disorders (MSD). MSD may lead to reduced productivity, decreased work ability, and even disability. The aim of this study was to describe the effects about occupational health medical team preventing and management of MSD. Method The design was prospective study describing the hightech industry workers screening and disability prevention for MSD. The quantitative analysis of the questionnaire was conducted through descriptive statistics and pair- $t$ test in order to indicate the direction and relationship between the two sets of occupational health medical team intervention program.

Results Of the 386 high-tech industry workers who completed the questionnaire. The use of pair-t test comparing two months of occupational health medical program, individual symptom scores significantly decreased 1.99 points to 6.12 points. The degree of functional subjects increased from $57 \%$ to $74 \%$, a significant improvement. Work ability index before treatment was 38.49 to 39.36 points after treatment improved, particularly in the self-evaluation and self-ability and physical work / effort needs very significant improvement in symptoms improve work ability index, increased efficiency and productivity. Subjects original degree of disability is about $22.33 \%$, significantly decreased to $18.1 \%$ after treatment.

Conclusions Early worksite screening and intervention for MSDs performed by occupational health medical team intervention program were effective on improving the work ability and the functional level. This service may also prevent worsening of the MSDs, and lead to significant reductions in occupational disorders, decreased health care costs, and improvements in production efficiency.

\section{WEEKEND WORK AND PSYCHOSOCIAL WELL-BEING IN KOREAN WORKERS}

${ }^{1}$ Hye-Eun Lee, ${ }^{1}$ Hyoung-Ryoul Kim, ${ }^{2}$ In-Ah Kim. ${ }^{1}$ Department of Occupational and Environmental Medicine, College of Medicine, The Catholic University of Korea, Seoul, Republic of Korea; ${ }^{2}$ Graduate School of Public Health, Yonsei University, Seoul, Republic of Korea

\subsection{6/oemed-2014-102362.288}

Objectives To identify association between weekend work and psychosocial well-being in a representative sample of Korean workers.

Method We analysed the associations between weekend work and psychosocial well-being in 29711 workers using data from the 2011 Korean Working Conditions Survey. Weekend work was defined by working one or more day on Saturday or on Sunday over the last month. Psychosocial well-being was measured by WHO well-being index. Multiple logistic regression analysis was performed adjusting age, education, income, regular/non-regular work, working time with stratifying sex and shift-work.

Results The prevalence of weekend work was higher in male $(62.4 \%)$ than in female $(54.8 \%)$. The longer working time per week, the more employees worked weekend $[<40$ (42.6\%), 40-48 (45.3\%), 49-60 (80.6\%), $\geq 61$ (94.9\%)]. Shift workers (87.3\%) worked more than non-shift workers $(56.2 \%)$ on weekend. In non-shift workers, weekend work group ( $\leq 4$ days) $[\mathrm{OR}=1.34$ (95\% CI 1.22-1.48), OR=1.17 (95\% CI 1.05-1.31)]and weekend work group ( $>4$ days) $[\mathrm{OR}=1.19$ (95\% CI 1.03-1.38), OR=1.30 (95\% CI 1.10-1.52)] were significant risk factors associated with lesser psychosocial well-being in male and female respectively.

Conclusions Weekend work is associated with a significant increase in lesser psychosocial well-being among Korean nonshift workers.

\section{EVALUATION OF SHIFT FATIGUE AND PHYSICAL HEALTH INTERVENTION IN PAPER MANUFACTURER OF WORKERS}

Ya-Yuan Hsu. Institute of Occupational Safety and Health, Taipei, Taiwan

10.1136/oemed-2014-102362.289

Objectives Shift and fatigue is one the most easily neglected health issues in occupation safety. The purposes of the study were to develop convenient method to evaluate the sources of fatigue in worksite and develop a physical health promotion program. Method The design was prospective study describing the paper manufacturer workers. Use myoton measuremented muscle stiffness and elasticity. The quantitative analysis of the three 
categories: managed, labour and repetitive motion types through descriptive statistics and two way ANOVA. Indicate the relationship between the two sets of 8 weeks of physical health intervention program.

Results Stress and lack of sleep were the sources of fatigue. The degree of discomfort in neck and shoulder and low back were related to the level of fatigue. There was a significant decrease in lower limb and back muscle stiffness and an increase in muscle elasticity measurements after physical health promotion program. Managed workers showed muscle tension is greater than the vertical type of labour.

Conclusions A well planned physical health program specifically designed the needs for the workforces can effectively change the perception of fatigue and reduce the level of muscle stiffness. Such promotion model can be further utilised in other occupational worksites.

\section{PREDICTING PHYSICIAN'S DUTY STRESS BY PARASYMPATHETIC NERVOUS FUNCTION (ALSO TO BE CONSIDERED FOR MINI-SYMPOSIUM: EARLY DETECTION AND MANAGEMENT OF WORKERS UNDER STRESS)}

${ }^{1,2}{ }^{2}$ Hsiu-Hao Lee, ${ }^{1}$ Bing-Yu Chen, ${ }^{2}$ Shih-Hsiang Lo, ${ }^{1}$ Pau-Chung Chen, 1,3Yue-Liang Guo. ${ }^{1}$ Institution of Occupational Medicine and Industrial Hygiene, National Taiwan University College of Public Health, Taipei, Taiwan; ${ }^{2}$ Department of Internal Medicine, Taipei City Hospital, Zhongxing Branch, Taipei, Taiwan; ${ }^{3}$ Department of Environmental and Occupational Medicine, National Taiwan University College of Medicine and NTU Hospital, Taipei, Taiwan

\subsection{6/oemed-2014-102362.290}

Objectives Early stress markers are useful in detecting workers with occupational stress. The aim of this study was to determine whether heart rate variability was associated with physicians' duty loading, and also a good predictor for stress markers.

Method An observational study on physicians with variable duty loading was conducted in a secondary referral medical centre in northern Taiwan in 2012. For every participant, 24-hr electrocardiography (EKG) and hourly blood pressure were obtained during three test days, i.e., regular-duty (only day shift), moderate-duty (day and night-shift with moderate number of patients cared), and high-duty days (day and night-shift with higher number of patients cared). Blood samples for stress markers were obtained at 8 am on the test day, and 8 am on the second morning.

Results A total of 12 staff physicians satisfactorily completed the study. The number of patients covered at night shift was $0,92 \pm$ 8 , and $187 \pm 9$, for regular-, moderate-, and high-duty nights, respectively. Total phone calls, urgent procedures, new patients admitted, critical patients cared and times of awakenings were significantly higher as the duty loads increased. The parasympathetic indicator derived from continuous EKG, high frequency normalised unit (HFnu), was negatively related to loading of total patient cared $(\mathrm{P}<0.0001)$. Reduced HFnu predicted elevated night systolic blood pressure $(\mathrm{P}=0.016)$ and serum uric acid $(\mathrm{P}=0.024)$, and $24 \mathrm{~h}$ urine vanillylmandelic acid $(\mathrm{P}=$ 0.0045), dopamine $(\mathrm{P}=0.011)$, and norepinephrine $(\mathrm{P}=$ 0.027).

Conclusions HFnu derived from heart rate variability measurement may predict several important stress markers during nightshift duties.

\section{DETERMINANTS OF OCCUPATIONAL HYGIENE EXPERT JUDGMENT ACCURACY}

${ }^{1}$ Mohammad Javad Zare Sakhvidi, ${ }^{1}$ Hamideh Mihanpoor, ${ }^{1}$ Abolfazl Barkhordari, ${ }^{2}$ Mehrdad Mostachaci. 'Department of Occupational Health, Faculty of Health, Shahid Sadoughi University of Medical Sciences, Yazd, Iran; ${ }^{2}$ Department of Occupational Medicine, Faculty of Medicine, Shahid Sadoughi University of Medical Sciences, Yazd, Iran

\subsection{6/oemed-2014-102362.291}

Objectives A cross sectional study was performed to determine the applicability and accuracy of expert judgment in occupational exposure assessment. The roles of educational session and determining factors were also realised.

Method Thirteen occupational hygienists were divided into two groups based on their field experience. They asked to evaluate exposure intensity in seven operating units in a tile factory before and after exposure training session. Participant's judgments were compared to actual air sampling data in the factory; and relative errors were calculated. Inter-class correlation coefficients were calculated and relative errors compared according to participants characteristics. Stepwise regressions were performed to investigate the defining variables.

Results In all situations there were almost perfect agreement (ICC >0.80) among raters. Correlations between estimated mean exposure and relative percentage error of participants before and after training were significant at 0.01 (correlation coefficients were -0.462 and -0.443 respectively). Results showed that actual concentration and experience resulted in $22.4 \%$ prediction variance for expert error as an independent variable.

Conclusions Correctness of exposure ratings by hygienists was susceptible to error from several sources. It seems that experienced subjects had better ability to predict the exposures. In general, in lower concentrations, the rating error increased significantly. Leading causes of judgment error should be taken into account in epidemiological exposure assessment studies.

\section{OCCUPATIONAL EXPOSURE AND STROKE - A CRITICAL REVIEW OF CHEMICAL AND PHYSICAL EXPOSURES}

1,2 $\mathrm{Per}$ Gustavsson, ${ }^{3}$ Kristina Jakobsson. ${ }^{1}$ Institute of Environmental Medicine, Karolinska Institutet, Stockholm, Sweden; ${ }^{2}$ Centre for Occupational and Environmental Medicine, Stockholm County Council, Stockholm, Sweden; ${ }^{3}$ Division of Occupational and Environmental Medicine, Lund University, Stockholm, Sweden

\subsection{6/oemed-2014-102362.292}

Objectives Stroke is the third most common cause of death in developed countries, exceeded only by coronary heart disease and cancer. There is substantial scientific literature on the association between occupational exposures and coronary heart disease, but much less is known about stroke. This systematic critical review was performed to assess the strength of evidence for causal associations between chemical and physical occupational exposures and stroke.

Method Literature on stroke incidence or mortality and occupational factors published up to 2012 was identified from Medline and Scopus. The 4471 abstracts were evaluated independently by two reviewers. 29 studies relevant to chemical and physical exposures were identified; ionising irradiation (7 studies); carbon disulfide (4), dynamite (3), motor exhaust (7) and other combustions products (8). The evidence for an association was assessed according to defined criteria as strong, moderate, limited, or insufficient. 